ESAIM: PROCEEDINGS AND SURVEYS, September 2014, Vol. 45, p. 128-137

J.-S. Dhersin, Editor

\title{
FINITE VOLUME SCHEME FOR ISOTROPIC KELLER-SEGEL MODEL WITH GENERAL SCALAR DIFFUSIVE FUNCTIONS*
}

\author{
Georges Chamoun $^{1,2}$, SaAd Mazen $^{1}$ and Talhouk RaAfat ${ }^{2}$
}

\begin{abstract}
This paper is devoted to the numerical analysis of a modified Keller-Segel model consisting of diffusion and chemotaxis with volume filling effect. Firstly, a finite volume scheme is generalized to the case of a Keller-Segel model allowing heterogeneities and discontinuities in the diffusion coefficients. For that, we start with the derivation of the discrete problem and then we establish a convergence result of the discrete solution to a weak solution of the continuous model. Finally, numerical tests illustrate the behavior of the solutions of this generalized numerical scheme.

Résumé. Cet article est consacré à l'analyse numérique d'un modèle généralisé de Keller-Segel modélisant l'interaction entre la densité des cellules et la concentration d'un chimio-attractant. La diffusion des cellules est modélisée par un opérateur dégénéré en évitant l'explosion de la densité cellulaire autour du chimio-attractant grâce au principe du "volume filling effect". D'abord, un schéma de volumes finis est généralisé au cas de modèles dégénérés de Keller-Segel avec des coefficients diffusifs hétérogènes discontinus. Ensuite, on montre la convergence des solutions du problème discret vers une solution faible du problème continu. Enfin, des tests numériques illustrent le comportement des solutions de ce schéma numérique généralisé.
\end{abstract}

\section{INTRODUCTION}

From microscopic bacteria through to the largest mammals, the survival of many organisms is dependent on their ability to navigate, through the detection of internal and external signals. The ability to migrate in response to chemical gradients, chemotaxis, has attracted significant interest due to its critical role in a wide range of biological phenomena. Mathematical modeling of chemotaxis has developed into a large and diverse discipline, whose aspects include its mechanistic basis and the modeling of specific systems. The Keller-Segel model of chemotaxis, introduced in [9] and [11], has provided a cornerstone for much of this work. A scheme recently developed in the finite volume framework (see [1]) treats the discretization of the Keller-Segel model in homogeneous domain where the diffusion tensor is considered to be the identity matrix. In this case, the mesh used for the discretization in space is assumed to satisfy the orthogonality condition (see [2]). In this paper, we propose and analyze the convergence of a generalized finite volume scheme applied to a degenerate chemotaxis model allowing heterogeneities and discontinuities in the diffusion coefficients. This generalization will help to reach more complex applications, especially that chemotaxis phenomenon (attraction or repulsion of cells via

* The authors would like to thank the National Council for Scientific Research (Lebanon), the Ecole Centrale de Nantes and the Lebanese University for their support to this work.

${ }^{1}$ Ecole centrale de Nantes, Laboratoire de Mathématiques Jean Leray and EDSTIM, CNRS UMR 6629, 1 rue de la Noé, 44321 Nantes, France.

georges.chamoun@ec-nantes.fr, mazen.saad@ec-nantes.fr

${ }^{2}$ Lebanese University, Laboratory of Mathematics-EDST and Faculty of Sciences I, Hadath, Liban. rtalhouk@ul.edu.lb

(C) EDP Sciences, SMAI 2014 
a chemo-attractant or a chemo-repellent) in a heterogeneous medium can yield such discontinuities, since the conductivities of the different components of the medium may be quite different.

\section{Setting of the Problem}

The well-known Keller-Segel model is introduced in general with homogeneous and isotropic diffusion (see [1]). In order to take into account the heterogeneities and the discontinuities in the diffusion coefficients, we consider the following coupled parabolic system:

$$
\begin{array}{r}
\partial_{t} u-\operatorname{div}(s(x) a(u) \nabla u)+\operatorname{div}(s(x) \chi(u) \nabla v)=0 \text { in } Q_{T}, \\
\partial_{t} v-\operatorname{div}(m(x) \nabla v)=g(u, v) \text { in } Q_{T},
\end{array}
$$

with the no-flux boundary conditions on $\Sigma_{t}=\partial \Omega \times(0, T)$,

$$
s(x) a(u) \nabla u \cdot \eta=0, m(x) \nabla v \cdot \eta=0,
$$

with initial conditions on $\Omega$,

$$
u(x, 0)=u_{0}(x), v(x, 0)=v_{0}(x) .
$$

We have $Q_{T}:=(0, T) \times \Omega$ where $T>0$ is a fixed time, and let $\Omega$ be a bounded domain in $\mathbb{R}^{d}, d=2$ or $d=3$ where the boundary $\partial \Omega$ is Lipschitz and $\eta$ is the unit outward normal vector.

The species $u$ represents the cell density and $v$ accounts for the chemical concentration. The diffusive flux modelling undirected (random) cell migration and the cross-diffusion flux with velocity dependent on the gradient of the signal, modelling the contribution of chemotaxis. Hillen and Painter were the first who introduced mechanistic descriptions of volume-filling effects (see [4]). Assuming that cells carry a certain finite (nonzero) volume and that occupation of an area limits other cells from penetrating it, a density-dependent chemotactic sensitivity $\chi(u)$ function, describing the probability of finding space by a local cell density $u$, can be derived. It models the migration of species $u$ to location with high concentration of $v$. The coefficient of diffusion is denoted by $a(u)$. The diffusive functions in a heterogeneous medium are denoted by $s(x)$ and $m(x)$ which may be discontinuous. The function $g(u, v)$ describes the rates of production and degradation of the chemical signal (chemoattractant); here, we assume it is of birth-death structure, i.e., a linear function,

$$
g(u, v)=\alpha u-\beta v ; \alpha, \beta \geq 0 .
$$

We assume that the density-dependent diffusion coefficient $a(u)$ degenerates for $u=0$ and $u=u_{m}$. This means that the diffusion vanishes when $u$ approaches values close to the threshold $u_{m}$ and also in the absence of cell-population. Secondly, we assume that $\chi(0)=0$ and there exists a maximum density of cells $u_{m}$ such that $\chi\left(u_{m}\right)=0$. The threshold condition has a clear biological interpretation; the cells stop to accumulate at a given point of $\Omega$ after their density attains certain threshold value $u_{m}$, therefore the chemotactical sensitivity $\chi(u)$ vanishes when $u$ tends to $u_{m}$. This interpretation is called the volume-filling effect, or prevention of overcrowding. The positivity of $\chi$ means that the chemical attracts the cells; the repellent case is the one of negative $\chi$.

Upon normalization of $u_{m}$, we can assume that $u_{m}=1$. Indeed, one can consider the following transformation: $\tilde{u}=\frac{u}{u_{m}}, \tilde{v}=v, \tilde{\chi}(\tilde{u})=\frac{\chi\left(\tilde{u} u_{m}\right)}{u_{m}}, \tilde{a}(\tilde{u})=a\left(\tilde{u} u_{m}\right), \tilde{g}(\tilde{u}, \tilde{v})=g\left(\tilde{u} u_{m}, \tilde{v}\right)$ and we omit the tildas in the notation. The main assumptions are:

$$
a:[0,1] \longmapsto \mathbb{R}^{+} \text {is continuous, } a(0)=a(1)=0 \text { and } a(q)>0 \text { for } 0<q<1 .
$$




$$
\chi:[0,1] \longmapsto \mathbb{R} \text { is continuous and } \chi(0)=\chi(1)=0 .
$$

A standard example for $\chi$ is:

$$
\chi(u)=u(1-u) \text { for } u \in[0,1] .
$$

Next, we require general diffusive functions $s$ and $m$ which may be discontinuous such that:

$$
s \in L^{\infty}(\Omega) \text { and there exists } \bar{s}, \underline{s} \in \mathbb{R}_{+}^{*} \text { such that } \underline{s} \leq s \leq \bar{s} \text { a.e } .
$$

Definition 2.1. Assume that $0 \leq u_{0} \leq 1, v_{0} \geq 0$ and $v_{0} \in L^{\infty}(\Omega)$. A weak solution of (1)-(4) is a pair $(u, v)$ of functions on $Q_{T}$ such that

$$
\begin{array}{r}
0 \leq u(x, t) \leq 1, v(x, t) \geq 0 \text { a.e. in } Q_{T}, \\
u \in L^{\infty}\left(Q_{T}\right), A(u):=\int_{0}^{u} a(r) d r \in L^{2}\left(0, T ; H^{1}(\Omega)\right), \\
v \in L^{\infty}\left(Q_{T}\right) \cap L^{2}\left(0, T ; H^{1}(\Omega)\right),
\end{array}
$$

and, for all $\varphi$ and $\psi \in \mathcal{D}([0, T) \times \bar{\Omega})$,

$$
\begin{array}{r}
-\int_{\Omega} u_{0}(x) \varphi(0, x) d x-\iint_{Q_{T}} u \partial_{t} \varphi d x d t+\iint_{Q_{T}} s(x) \nabla A(u) \cdot \nabla \varphi d x d t-\iint_{Q_{T}} s(x) \chi(u) \nabla v \cdot \nabla \varphi d x d t=0, \\
-\int_{\Omega} v_{0}(x) \psi(0, x) d x-\iint_{Q_{T}} v \partial_{t} \psi d x d t+\iint_{Q_{T}} m(x) \nabla v \cdot \nabla \psi d x d t=\iint_{Q_{T}} g(u, v) \psi d x d t .
\end{array}
$$

\section{NUMERICAL SCHEME}

This section is devoted to the formulation and to the proof of convergence of a finite volume scheme for a Keller-Segel model with general isotropic scalar diffusion functions. We will first describe the space and time discretizations, then we will give the numerical scheme and the result of convergence.

\subsection{Space And Time Discretizations.}

The space discretization of the domain $\Omega$ is based on an admissible mesh as defined in [2] (see Figure 1). It is a finite family $\mathcal{T}_{h}$ of polygonal open convex subsets $K$ of $\Omega$, called the control volumes such that $\bar{\Omega}=\cup_{K \in \mathcal{T}_{h}} \bar{K}$, where $h=\sup _{K \in \mathcal{T}_{h}}(\operatorname{diam}(K))$, together with a finite family $\mathcal{E}$ of disjoint subsets of $\bar{\Omega}$ consisting in non-empty open convex subsets $\sigma$ of affine hyperplanes of $\mathbb{R}^{d}$, called the edges, and a family $\mathcal{P}=\left\{x_{K}, K \in \mathcal{T}_{h}\right\}$ of points in $\Omega$, called the centers verifying the following properties,

- For any edge $\sigma \in \mathcal{E}$, either $\sigma \subset \partial \Omega$ or $\sigma=\bar{K} \cap \bar{L}$ for some $K \neq L$ in $\mathcal{T}_{h}$. In the latter case, we denote $\sigma=\sigma_{K, L}$, called the interfaces. We denote by $N(K)$ the control volumes neighbors of $K$.

- For any $K \in \mathcal{T}_{h}$, the point $x_{K}$ belongs to $K$. For any $\sigma_{K, L} \in \mathcal{E}$, the line $\left(x_{K}, x_{L}\right)$ is orthogonal to $\sigma_{K, L}$. In addition to that, for any interior edge $\sigma_{K, L}$, we denote by $n_{K, L}$ and $d_{K, L}$, respectively, the unit vector normal to $\sigma_{K, L}$ outward of $K$ and the distance from $x_{K}$ to $x_{L}$. For any exterior edge $\sigma$, the distance is taken from the center $x_{K}$ to the middle point of the edge $\sigma$. The measure of $K$ is denoted by $|K|$ and the $(d-1)$-dimensional measure of $\sigma \in \mathcal{E}$ is denoted by $|\sigma|$.

The time discretization is the sequence of discrete times $t^{n}=n \Delta t$ for $n \in \mathbb{N}$, where $\Delta t>0$ is a given time-step.

Let us consider $\mathcal{T}_{h}$ as an admissible mesh, such that the discontinuities of $s$ and $m$ belongs to the interfaces of this mesh. The numerical scheme is obtained by using the finite volume method: equations (1) and (2) of the model are integrated on each control volume $K$ and interval of time $\left(t^{n}, t^{n+1}\right)$ and then we shall approximate 


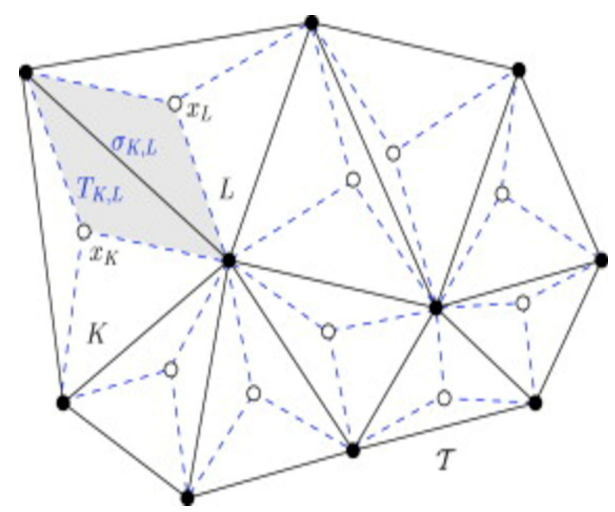

Figure 1. A space discretization of $\Omega$.

the normal fluxes over each edge $\sigma$ of $K$. Note that if $s(x)=1$, the approximation of the normal diffusive flux $\nabla A(u) \cdot \eta_{K, L}$ on the interface $\sigma=\sigma_{K, L}$ has been detailed in [1] and the transmissibilities were defined as $\tau_{K, L}=\frac{\left|\sigma_{K, L}\right|}{d_{K, L}}$. In the following subsections, we will construct an approximation of the normal diffusive flux $s(x) \nabla A(u) \cdot \eta_{K, L}$ with continuous and discontinuous heterogeneous diffusive functions $s(x)$.

\subsection{Continuous Diffusion Coefficients.}

In this case, the admissibility assumption on $\mathcal{T}_{h}$ allows us to simply approximate this normal diffusive flux by means of the divided differences,

$$
\int_{\sigma_{K, L}} s(x) \nabla A(u) \cdot \eta_{K, L} \approx s_{K, L} \frac{\left|\sigma_{K, L}\right|}{d_{K, L}}\left(A\left(u_{L}\right)-A\left(u_{K}\right)\right)
$$

where $s_{K, L}$ denotes the approximation of $s(x)$ on the interface $\sigma_{K, L}$, with $s_{K, L}:=s\left(\bar{x}_{K, L}\right)$ such that $\bar{x}_{K, L}$ is the intersection between the segment $\left[x_{K}, x_{L}\right]$ and the common interface $\sigma_{K, L}$. Consequently, the new transmissiblities in this case are:

$$
\tau_{K, L}=s_{K, L} \frac{\left|\sigma_{K, L}\right|}{d_{K, L}}
$$

\subsection{Discontinuous Diffusion Coefficients.}

In order to treat the case of discontinuities of the diffusion coefficients which lay over the boundaries of the control volumes, let us introduce,

$$
s_{K}=\frac{1}{|K|} \int_{K} s(x) d x \text { and } s_{K, \sigma}=\left|s_{K} \eta_{K, \sigma}\right|,
$$

where |.| is the Euclidean norm, $s_{K, \sigma}$ be the approximation of $s(x)$ on the edge $\sigma=\sigma_{K, L}$ and $\eta_{K, \sigma}$ is the unit outward normal vector at $\sigma$ with respect to $K$.

In order to obtain the local conservativity, we will introduce auxiliary unknowns $u_{\sigma}$ on the interfaces. These auxiliary unknowns are helpful to write the scheme, but they can be eliminated locally so that the discrete equations will only be written with respect to the primary unknowns $\left(u_{K}\right)_{K \in \mathcal{T}_{h}}$. Since $s$ is continuous on the interior of $K$ and $L$, the approximation $H_{\sigma}$ of the diffusive flux $s(x) \nabla A(u) \cdot \eta_{K, L}$ may be performed on each 
side of $\sigma=\sigma_{K, L}$ by using the finite difference principle,

$$
H_{\sigma}=s_{K, \sigma} \frac{A\left(u_{\sigma}\right)-A\left(u_{K}\right)}{d_{K, \sigma}} \text { on } K ; H_{\sigma}=s_{L, \sigma} \frac{A\left(u_{L}\right)-A\left(u_{\sigma}\right)}{d_{L, \sigma}} \text { on } L,
$$

where $d_{K, \sigma}$ (resp. $d_{L, \sigma}$ ) is the distance from $x_{K}$ (resp. $x_{L}$ ) to the interface $\sigma_{K, L}$. Requiring the two above approximations to be equal (the conservativity of the diffusive flux) yields the value of $A\left(u_{\sigma}\right)$,

$$
A\left(u_{\sigma}\right)=\frac{1}{\frac{s_{L, \sigma}}{d_{L, \sigma}}+\frac{s_{K, \sigma}}{d_{K, \sigma}}}\left(A\left(U_{L}\right) \frac{s_{L, \sigma}}{d_{L, \sigma}}+A\left(U_{K}\right) \frac{s_{K, \sigma}}{d_{K, \sigma}}\right) .
$$

This latter allows to give the expression of the approximation $H_{\sigma}$,

$$
H_{\sigma}=\tau_{\sigma}\left(A\left(U_{L}\right)-A\left(U_{K}\right)\right) \text { with } \tau_{\sigma}=\frac{s_{K, \sigma} s_{L, \sigma}}{s_{L, \sigma} d_{K, \sigma}+s_{K, \sigma} d_{L, \sigma}} .
$$

Consequently,

$$
\int_{\sigma_{K \mid L}} s(x) \nabla A(u) \cdot \eta_{K, L} \approx \tau_{\sigma}\left|\sigma_{K, L}\right|\left(A\left(u_{L}\right)-A\left(u_{K}\right)\right)
$$

with the following new transmissibilities:

$$
\tau_{K, L}=\tau_{\sigma}\left|\sigma_{K, L}\right|
$$

The same guidelines are used to obtain an approximation $G_{\sigma}$ of the flux $m(x) \nabla v \cdot \eta_{K, L}$.

\subsection{Numerical Scheme.}

In addition to the previous subsections, we still have to approximate $s(x) \chi(u) \nabla v \cdot \eta_{K, L}$ by means of the values $U_{K}, U_{L}$ and $\delta V_{K, L}$ (where $\delta V_{K, L}$ denotes the approximation of the flux $s(x) \nabla v \cdot \eta_{K, L}$ on $\sigma_{K, L}$ ) that are available in the neighborhood of the interface $\sigma_{K, L}$. To do this, we use a numerical flux function $G\left(U_{K}, U_{L}, \delta V_{K, L}\right)$. Numerical convection flux functions $G$ of arguments $(a, b, c) \in \mathbb{R}^{3}$, are required as in [1] to satisfy the following properties,

- $G(., b, c)$ is non-decreasing for all $b, c \in \mathbb{R}$, and $G(a, ., c)$ is non-increasing for all $a, c \in \mathbb{R}$;

- $G(a, b, c)=-G(b, a,-c)$ for all $a, b, c \in \mathbb{R}$; hence the flux is conservative.

- $G(a, a, c)=\chi(a) c$ for all $a, c \in \mathbb{R}$; hence the flux is consistent.

- There exists $C>0$, such that for all $a, b, c \in \mathbb{R},|G(a, b, c)| \leq C(|a|+|b|)|c|$.

- $\left|G(a, b, c)-G\left(a^{\prime}, b^{\prime}, c\right)\right| \leq|c|\left(\left|a-a^{\prime}\right|+\left|b-b^{\prime}\right|\right)$ for all $a, b, a^{\prime}, b^{\prime}, c \in \mathbb{R}$.

Remark 3.1. One possibility to construct the numerical flux $G$ is to split $\chi$ in the non-decreasing part $\chi_{\uparrow}$ and the non-increasing part $\chi_{\downarrow}$, such that

$$
\chi_{\uparrow}(z):=\int_{0}^{z}\left(\chi^{\prime}(s)\right)^{+} d s, \chi_{\downarrow}(z):=\int_{0}^{z}\left(\chi^{\prime}(s)\right)^{-} d s .
$$

Herein $s^{+}=\max (s, 0)$ and $s^{-}=\max (-s, 0)$. Then we take,

$$
G(a, b, c)=c^{+}\left(\chi_{\uparrow}(a)+\chi_{\downarrow}(b)\right)-c^{-}\left(\chi_{\uparrow}(b)+\chi_{\downarrow}(a)\right) .
$$

Notice that in the case $\chi$ has unique local (and global) maximum at the point $\bar{u} \in[0,1]$, such as the flux $\chi(u)=u(1-u)$, we have

$$
\chi_{\uparrow}(z)=\chi(\min \{z, \bar{u}\}) \text { and } \chi_{\downarrow}(z)=\chi(\max \{z, \bar{u}\})-\chi(\bar{u}) .
$$



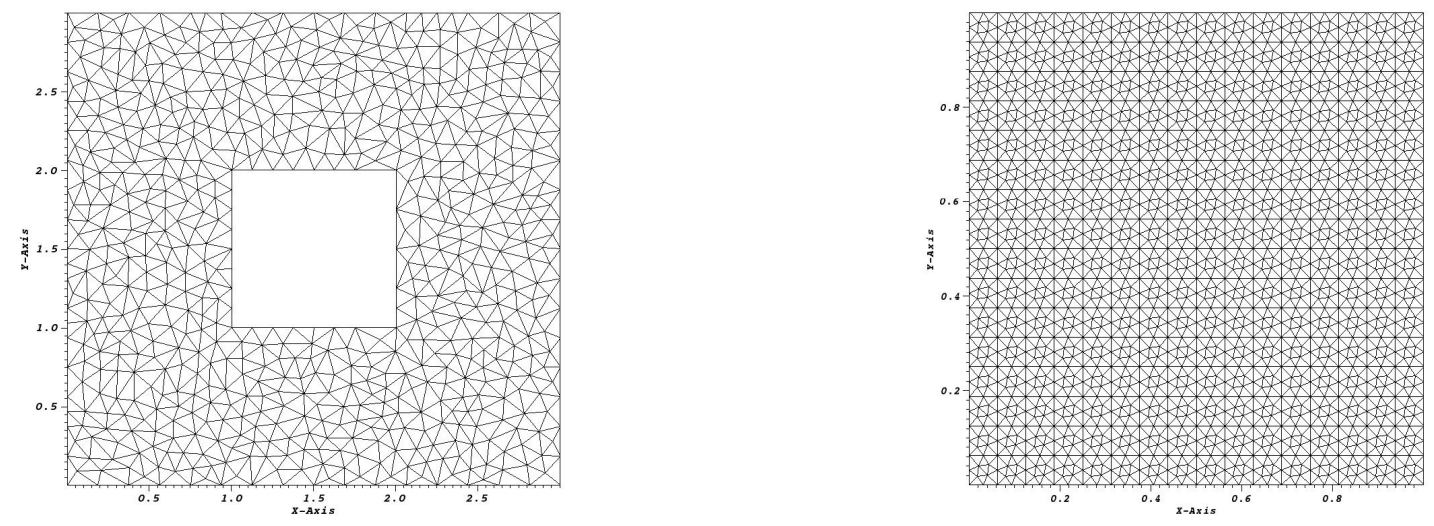

FIgURE 2. An admissible triangulations $\mathcal{T}_{h}$ : Mesh of 1253 triangles with an empty hall (left) and of 3584 triangles (right) .

Finally, we obtain the following scheme: $\forall K \in \mathcal{T}_{h}$,

$$
U_{K}^{0}=\frac{1}{|K|} \int_{K} u_{0}(x) d x, V_{K}^{0}=\frac{1}{|K|} \int_{K} v_{0}(x) d x
$$

and $\forall n \in[0, \ldots, N]$,

$$
\begin{gathered}
|K| \frac{U_{K}^{n+1}-U_{K}^{n}}{\Delta t}-\sum_{L \in N(K)} \tau_{K \mid L}\left(A\left(U_{L}^{n+1}\right)-A\left(U_{K}^{n+1}\right)\right)+\sum_{L \in N(K)} G\left(U_{K}^{n+1}, U_{L}^{n+1} ; \delta V_{K, L}^{n+1}\right)=0, \\
|K| \frac{V_{K}^{n+1}-V_{K}^{n}}{\Delta t}-\sum_{L \in N(K)} \mu_{K, L}\left(V_{L}^{n+1}-V_{K}^{n+1}\right)=|K| g\left(U_{K}^{n}, V_{K}^{n+1}\right),
\end{gathered}
$$

where $\delta V_{K, L}^{n+1}=\tau_{K \mid L}\left(V_{L}^{n+1}-V_{K}^{n+1}\right), \tau_{K, L}$ (resp. $\left.\mu_{K, L}\right)$ are the new transmissibilities defined in (10) (or (13)) and the unknowns are $U=\left(U_{K}^{n+1}\right)_{K \in \mathcal{T}_{h}}$ and $V=\left(V_{K}^{n+1}\right)_{K \in \mathcal{T}_{h}}, n \in[0 . . N]$. The discrete solution associated to this discrete problem is, $\left(u_{h}, v_{h}\right)$ defined as constant functions on $Q_{T}$ given by,

$$
\forall K \in \mathcal{T}_{h}, \forall n \in[0, \ldots, N],\left.u_{h}\right|_{] t^{n}, t^{n+1}\right] \times K}=U_{K}^{n+1},\left.v_{h}\right|_{] t^{n}, t^{n+1}\right] \times K}=V_{K}^{n+1} .
$$

For this numerical scheme, we have proved the following result.

Theorem 3.1. Assume (5), (7), (6) and (8). Consider $v_{0} \in L^{\infty}(\Omega), v_{0} \geq 0$ and $0 \leq u_{0} \leq 1$ a.e. on $\Omega$.

1) There exists a solution $\left(u_{h}, v_{h}\right)$ of the discrete system (16)-(17) with initial data (15).

2) Any sequence $\left(h_{m}\right)_{m}$ decreasing to zero possesses a subsequence, still denoted as the sequence, such that $\left(u_{h_{m}}, v_{h_{m}}\right)$ converges a.e. on $Q_{T}$ to a weak solution $(u, v)$ of the modified Keller-Segel system (1)-(4) in the sense of Definition 2.1.

Outline of the Proof. The discrete maximum principle still verified due to the positivity of the new transmissibilities defined in (10) and in (13). Consequently, one can maintain the same necessary estimates of [1] to prove the existence of a discrete solution. Then, estimates in time and space are constructed to use the Kolmogorov compactness criterion and to conclude the existence of a subsequence $\left(u_{h}, v_{h}\right)$ of discrete solutions that converges to a function $(u, v)$ almost everywhere in $[0, T] \times \Omega$. Finally, we proved that this function $(u, v)$ is a weak solution of the modified Keller-Segel model. 

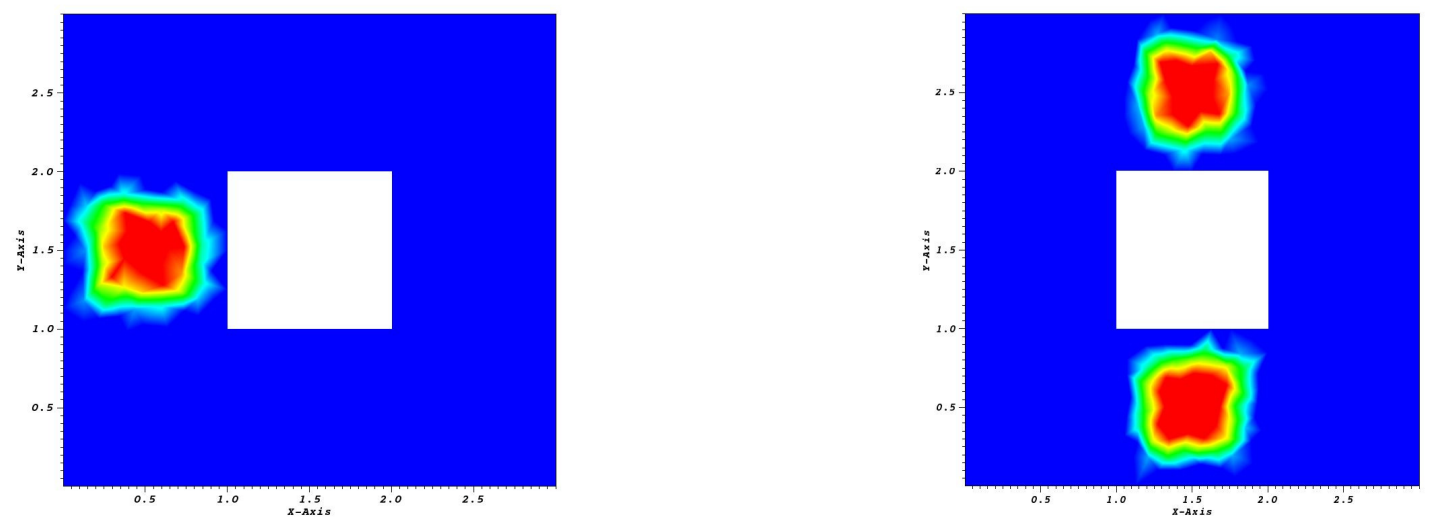

Figure 3. Test 1- Initial conditions for the cell density $u_{0}$ (left) and for the concentration of the chemo-attractant $v_{0}$ (right) .
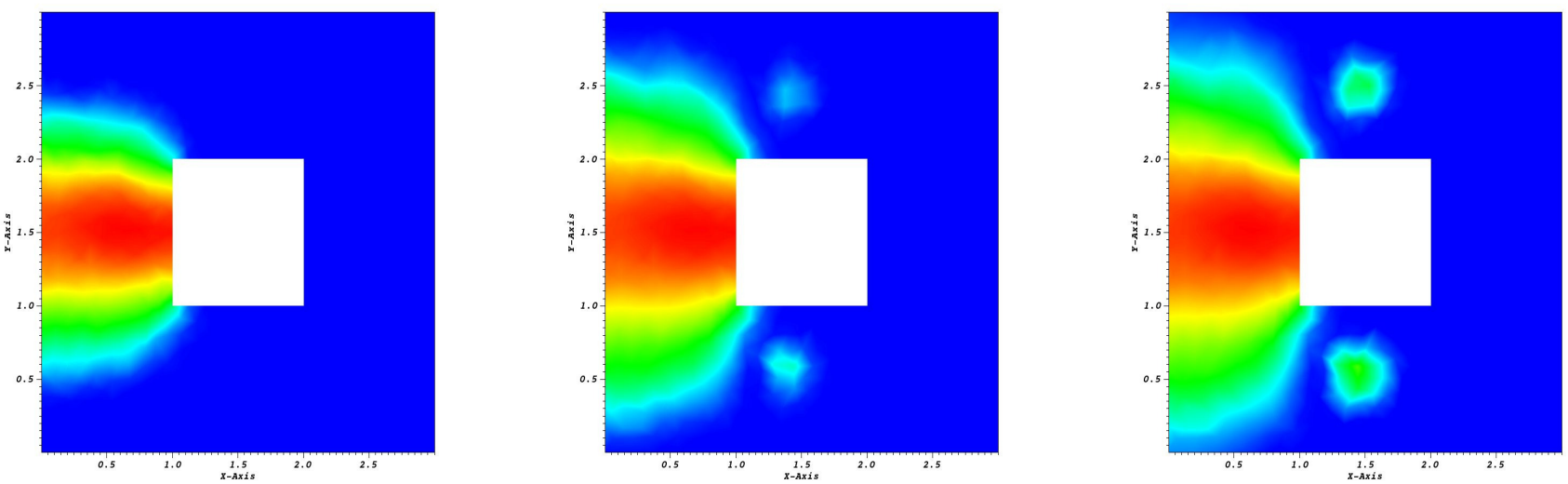

Figure 4. Test 1 - Evolution of the cell density $(u)$, at time $t=5$ with $0 \leq u \leq 0.3283$ (left), at time $t=12.5$ with $0 \leq u \leq 0.2413$ and at time $t=17.5$ with $0 \leq u \leq 0.2150$ (right) .

\section{NumericAl TESTS}

In this section, we shall illustrate the behavior of the discrete solutions of the proposed numerical scheme for isotropic heterogeneous and discontinuous coefficients. The computations were done with numerical handwork Fortran 95 code, where this scheme is implemented. The algorithm used to compute numerical solution of the discrete problem is the following: at each time step, we first calculate $V^{n+1}$ solution of the linear system given by the equation of (17) and next we compute $U^{n+1}$ as the solution of the nonlinear system defined by the first equation of (16). For this end, a Newton algorithm is implemented to approach the solution of nonlinear system and a bigradient method to solve linear systems arising from the Newton algorithm process. We will provide our tests on admissible meshes given in the Figure 2. 

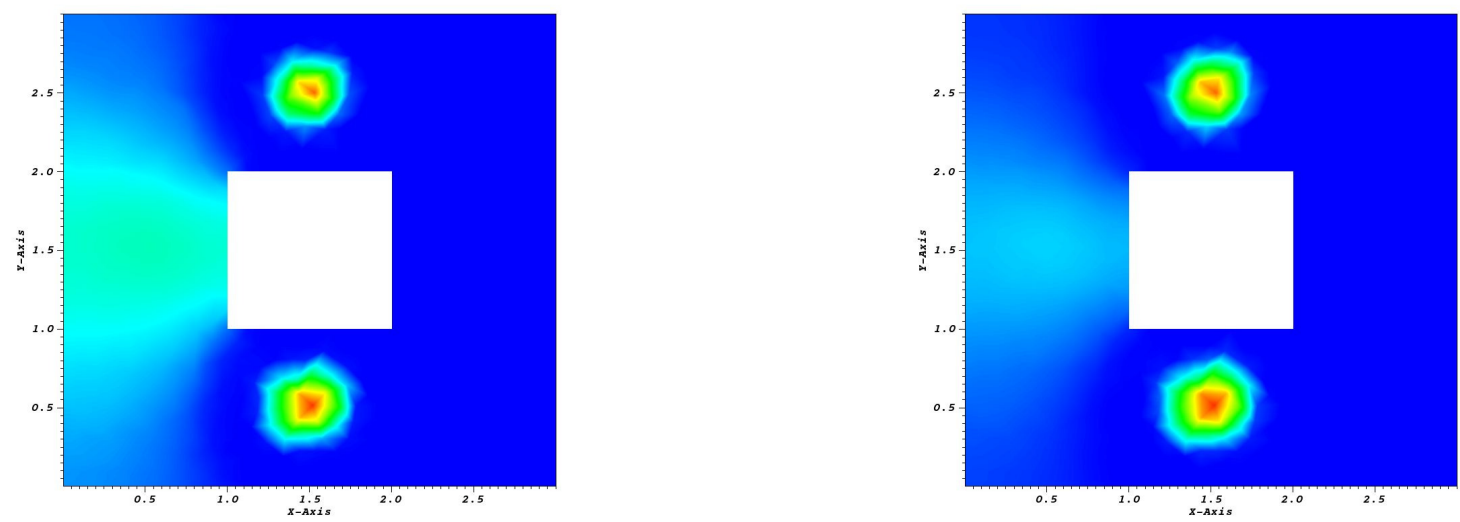

Figure 5. Test 1 - The cell density $(u)$ at time $t=50$ with $0 \leq u \leq 0.4973$ (left), at time $t=100$ with $0 \leq u \leq 0.7047$ (right) .
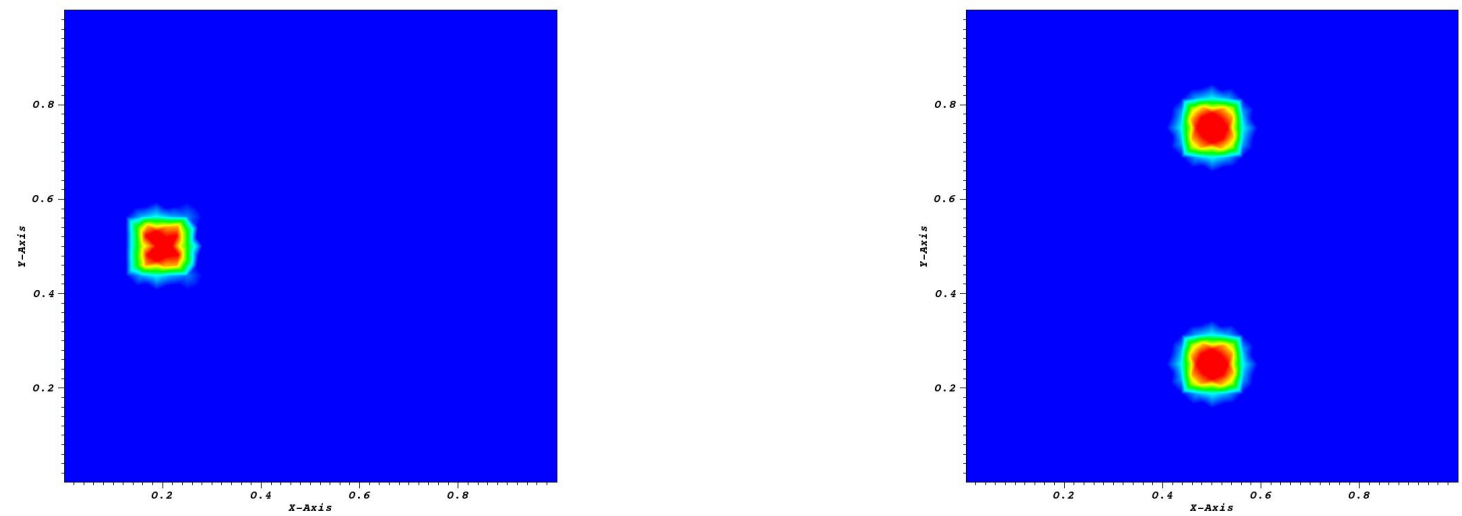

Figure 6. Test 2- Initial conditions for the cell density $u_{0}$ (left) and for the concentration of the chemo-attractant $v_{0}$ (right) .

Test 1: Continuous heterogeneous case. Let us consider the following data: $L_{x}=3, L_{y}=3$ as the length and the width of the domain given in the Figure 1 (left). In this first test, we consider the diffusion functions as

$$
s(x)=(x-1.5)^{2}+(y-1.5)^{2} ; m(x)=1 .
$$

Further, $d t=0.005, \alpha=0.01, \beta=5 \times 10^{-5}, A(u)=D\left(\frac{u^{2}}{2}-\frac{u^{3}}{3}\right)$, with $D=10^{-1}, \chi(u)=c u(1-u)^{2}$, with $c=10^{-1}$. Finally, the diffusion coefficient of the chemo-attractant is $d=10^{-4}$. The initial conditions are defined by regions. The initial density is defined as $u_{0}(x, y)=1$ in the square $(x, y) \in([0.2,0.8] \times[1.2,1.8])$ and 0 otherwise. The initial chemoattractant is defined as $v_{0}(x, y)=5$ in the union of two squares $(x, y) \in$ $([1.2,1.8] \times[0.2,0.8]) \cup([1.2,1.8] \times[2.2,2.8])$ and 0 otherwise (see Figure 3). In Figures 4 and 5, we show the evolution of the cell density. We observe during the stage of evolution the effect of the chemo-attractant, since the cells are present in the chemoattractant regions. 

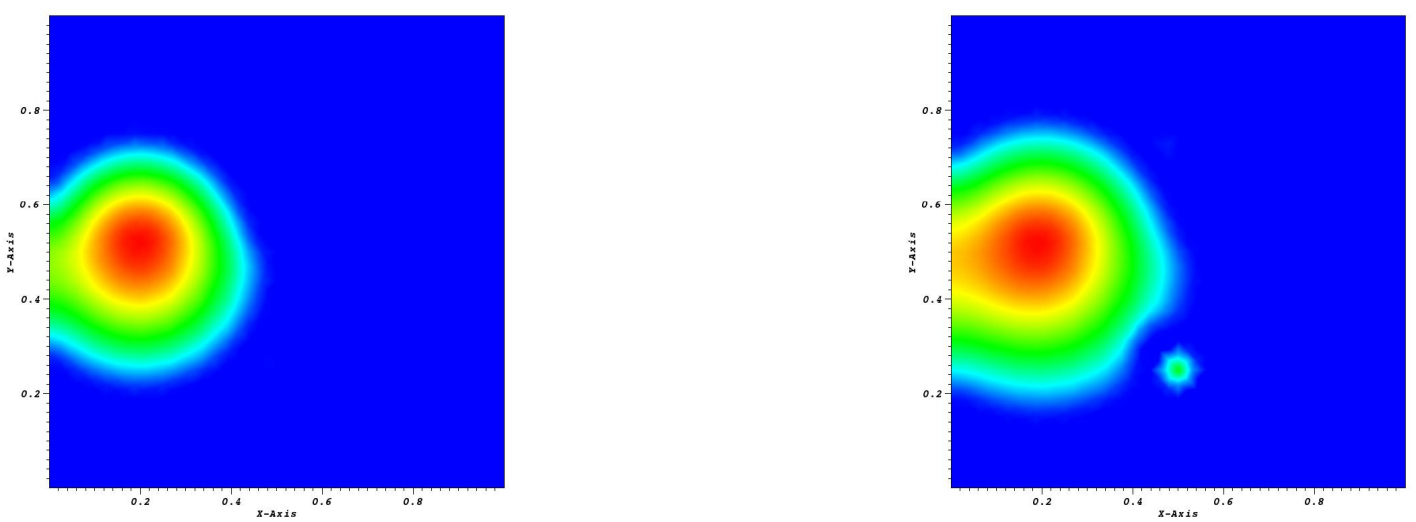

Figure 7 . Test 2 - The cell density $(u)$ at time $t=2.5$ with $0 \leq u \leq 0.1055$ (left) and at time $t=5$ with $0 \leq u \leq 0.07963$ (right) .
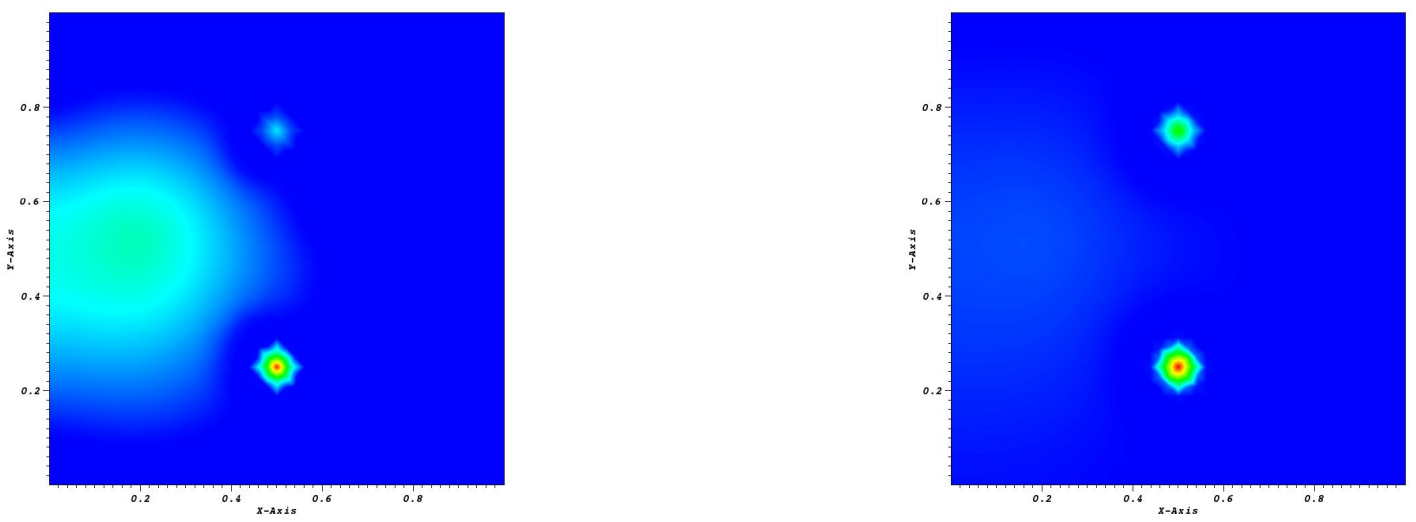

Figure 8 . Test 2 - The cell density $(u)$ at time $t=10$ with $0 \leq u \leq 0.1991$ (left) and at time $t=40$ with $0 \leq u \leq 0.5570$ (right) .

Test 2: Discontinuous case. In this test, our target is to prove the efficiency of our numerical scheme in treating discontinuous diffusion. For that, let us consider

$$
s(x)=\left\{\begin{array}{ll}
6 & \text { if } y \leq 0.5 \\
1 & \text { if } y>0.5
\end{array}, m(x)=1\right.
$$

The space domain $\Omega$ is the unit square and the mesh is given in the Figure 1 (right). One can remark that the discontinuities of the diffusion coefficients coincide with the interfaces of the mesh. Further, $d t=0.005, \alpha=0.01$, $\beta=0.05, A(u)=D\left(\frac{u^{2}}{2}-\frac{u^{3}}{3}\right)$ with $D=0.03, \chi(u)=c u(1-u)^{2}$ with $c=0.1$. Finally, the diffusion coefficient of the chemo-attractant is $d=10^{-5}$. The initial conditions are also defined by regions. The initial density is defined as $u_{0}(x, y)=1$ in the square $(x, y) \in([0.15,0.25] \times[0.45,0.55])$ and 0 otherwise. The initial chemoattractant is defined as $v_{0}(x, y)=10$ in the union of two squares $(x, y) \in([0.45,0.55] \times[0.7,0.8]) \cup([0.45,0.55] \times[0.2,0.3])$ and 0 otherwise (see Figure 6 ). The behavior of the cell density via the chemo-attractant and the influence of the 
discontinuous diffusion coefficients are clear, as can be seen in the Figures 7 and 8 . We observe during the stage of evolution the effect of the anisotropic diffusion since the cells are more present in the down chemoattractant region.

\section{CONCLUSION}

In this article, we propose a variant of the Keller-Segel model and a finite volume numerical method to simulate this chemotaxis model with general scalar diffusion functions. The approximate solutions remains biologically admissible due to the confinement of the cell density as a consequence of the discrete maximum principle. The convergence to a weak solution of the continuous model is guaranteed and the numerical experiments allow the validation of the generalized numerical scheme.

\section{REFERENCES}

[1] B. Andreianov, M. Bendahmane and M. Saad, Finite volume methods for degenerate chemotaxis model. Journal of computational and applied mathematics, 235: p. 4015-4031, 2011.

[2] R. Eymard, T. Gallouet and R. Herbin, Finite volume methods, Handbook of numerical analysis. Handbook of numerical analysis, Vol VII North-Holland, Amsterdam, p. 713-1020, 2000.

[3] K. Brenner, Méthode de volumes finis sur maillages quelconques pour des systèmes d'évolution non linéaires. Thèse soutenue à l'Université Paris XI, le 8 novembre 2011.

[4] T. Hillen and K. Painter, A user's guide to PDE models for chemotaxis. J Math Biol.; 58(1-2), p. 183-217, 2009.

[5] R. Herbin and F. Hubert, Benchmark on discretization schemes for anisotropic diffusion problems on general grids. 5th International Swnposium on Finite Volumes For Complex Applications, 2008.

[6] R. Eymard, T. Gallouet, R. Herbin and J.C. Latche, Analysis tools for finite volume schemes. Acta Math. Univ. Comenianae, Vol. LXXVI, 1, p. 111-136, 2007.

[7] J.L. Lions, Quelques méthodes de résolution des problèmes aux limites non-linéaires. Dunod, Paris, 1969.

[8] B. Dibenedetto, Degenerate Parabolic Equations. Springer-Verlag, New York, 1993.

[9] D. Horstmann, from 1970 until present, The keller-Segel model in chemotaxis and its consequences. I.Jahresberichte DMV 105 (3), p. 103-165, 2003.

[10] R. Eymard, T. Gallouet, R. Herbin and A. Michel, Convergence of a finite volume scheme for nonlinear degenerate parabolic equations. Numer .Math. 92(1), p. 41-82, 2002.

[11] E.F. Keller and L.A. Segel, The Keller-Segel model of chemotaxis. J Theor Biol. 26, p. 399-415, 1970.

[12] H. Brezis, Analyse fonctionnelle, Théorie et Applications, Masson, Paris, 1983. 